\title{
Pulsatile Laminar Flows in a Dilated Channel Using Cartesian Curvilinear Coordinates
}

\author{
Sumaia Parveen Shupti, Rumia Sultana, Mir Golam Rabby, Md. Mamun Molla*
}

Dept. of Electrical Engineering and Computer Science, North South University, Dhaka-1229, Bangladesh

*Corresponding Author: mmamun@northsouth.edu, mmamun@gmail.com

Copyright (C)2013 Horizon Research Publishing All rights reserved.

\begin{abstract}
In this paper, numerical simulation has been performed to study the flow behavior of a pulsatile Newtonian fluid confined within a two-dimensional (2D) channel with an asymmetric shaped aneurysm using the finite volume method. The governing NavierStokes equations have been modified using the Cartesian curvilinear coordinates to handle complex geometries. The investigation have been carried out to characterize the blood flow for Reynolds number 100, 300 and 500 which is suitable for human artery. The numerical results are represented here in terms of velocity, pressure distribution, wall shear stress as well as the streamlines indicating the recirculation zones inside the dilated region. The results of this study demonstrate a lower centerline velocity inside the aneurysm but comparatively higher wall pressure and wall shear stress at the distal neck of the aneurysm. Furthermore, all three Reynolds numbers show the presence of vortex inside the bulge.
\end{abstract}

Keywords Aneurysm, finite volume method, laminar pulsatile flow, wall shear stress, recirculation zone

\section{Introduction}

Abdominal aortic aneurysm (AAA) is the abnormal dilation of abdominal aorta due to weakening of the wall. Since the cause and nature of AAAs is an important matter of debate, it can only be said that aneurysm formation depends on the interplay between factors that either weaken the wall or increase the load on it [10]. Untreated aneurysm may burst under insistent internal pressure causing death or disability [7]. Again, the foremost health danger of an AAA is rupture which leads to death in up to $90 \%$ of the victims making AAAs the 15th leading cause of death in the United States [1].

Since the internal mechanical forces are initiated by the dynamic action of blood flow inside the aneurysm, studying the hemodynamic factors might indicate the risk caused by AAA [4]. Several experiments and numerical investigations of pulsatile flow have been performed during recent times to study the hemodynamic behavior. The numerical simulation in the arterial aneurysm was initially done by Perktold [12] who investigated the pul- satile blood flow by introducing the paths of single particles in a model axisymmetric aneurysm. The simulation showed the development, shift and disappearance of vortices in an arterial segment with an aneurysm and also the stagnation zones. Later, Kumar and Naidu [7] numerically analyzed the nonlinear axisymmetric pulsatile blood flow dynamics in rigid vessels with varying degrees of dilation using a transient velocity-pressure finite element method (FEM). The result showed time-varying recirculation regions which can damage the blood cells and also high shear stresses near the ends of aneurysm.

The blood flow behavior in a two-aneurysm model was numerically predicted by Finol and Amon [3] where they used the spectral element method under pulsatile conditions for the range of time-averaged Reynolds numbers $50 \leq R e_{m} \leq 300$. They found a very low wall shear stress at the center of the bulge region and high wall shear stress gradients in the distal end of each bulge by tracing the vortex dynamics. They concluded with higher oscillating stresses for large aneurysms and lower for small ones at the same time-averaged Reynolds number. Almost similar result was drawn by Kumar [8] in his space-time analysis of pulsatile flow dynamics in an arterial vessel with multiple aneurysms in series based on a time accurate cell centered finite volume scheme in implicit Euler time marching setting. He investigated the influence of Reynolds number (Re), Strouhal's number (St), and degree of dilation and found high wall shear stresses and high wall pressures specially at the distal end of the large aneurysms.

The first mathematical model to couple both the growth and the rupture of the AAA was presented by Volokh and Vorp [17] where they provided reasonable qualitative results showing possible AAA ruptures. Swillens et al. [14] constructed AAA geometry with the help of patient specific CT (Computed Tomography) -scans and employed both experimental and numerical methods to investigate the influence of an AAA on hemodynamics. They found that the presence of AAA significantly alters wave reflection and hemodynamics in the aorta with apparently measurable effects in human body.

The flow in aneurysms with simple ring-shaped stent obstacles was simulated by $\mathrm{Xu}$ and Lee [19] who examined the flow inside the aneurysm. They used a Lat- 
tice Boltzmann Method (LBM) with an extrapolation boundary technique and developed a scientific programming strategy based on object-oriented concepts to ease the code development. Multiphase non-Newtonian fluid simulations of pulsatile flow were performed and compared with the standard Newtonian fluid models by Kim et al. [6] to investigate the influence of mechanical factors on the hemodynamics and identify critical factors of non-Newtonian models. They also identified the multiphase non-Newtonian viscosity model as an optimal one to demonstrate the effects of changes in hematocrit.

$\mathrm{Li}[9]$ conducted a follow-up study that included fortyfour patients with AAAs to investigate wall stress. Abdominal ultra sonography and CT scans were performed and patient-specific 3-dimensional AAA geometries were constructed from the CT images. From this study he found the presence of high stress at the shoulder region of the aneurysm. Decreasing stress was also found with increasing diameter of the bulge at the shoulder. A CFD simulations in realistic models of cerebral aneurysms was described by Valencia et al. [16]. Twenty lateral aneurysm models obtained from three-dimensional rotational angiographic imaging data were investigated. The WSS on the aneurysm sac of the 20 investigated aneurysms were different than each other. They concluded that a measure of the geometrical parameter NSI (Non Sphericity Index) could help to predict rupture risk of the cerebral aneurysm in the patient and therefore the urgency of intervention.

Yip and $\mathrm{Yu}[20]$ investigated the transition to turbulence in pulsatile AAA flows. Sine waveforms were used to simulate aortic flow conditions for time averaged Reynolds number $R e_{\text {mean }}=1600-2100$ and Wolmersley parameter $\alpha=7.2-12.2$. They found that instability grows progressively during the acceleration phase and transition to turbulence in the bulge occurs shortly after the commencement of the deceleration phase. Farnoush et al. [2] investigated the hemodynamic effects resulting from a change in the parent artery diameter of bifurcation type aneurysm. Calculations were performed with steady flow rate $(125 \pm 12.5 \mathrm{ml} / \mathrm{min})$ at the parent artery inlet and the Energy Loss (EL) was calculated from pressure and kinetic energy obtained from flow velocity. The results showed high WSS and EL in the model with the smallest parent vessel.

The purpose of this work is to study elaborately the hemodynamic factors that generally contribute to the rupture of AAAs i.e., the centerline velocity, wall pressure, wall shear stress, streamlines, vortex formation during a cardiac cycle, patterns of wall shear stresses through different phases of a cardiac cycle and also variations of velocity and pressure in time with the help of numerical simulation technique to achieve very good flow insight in an asymmetric aneurysm.

\section{Computational Modeling}

Incompressible, homogeneous and sinusoidal pulsatile flow is simulated for the Reynolds numbers 100, 300 and 500. The arterial wall is assumed rigid and blood is modeled as a Newtonian fluid with a density, $\rho=$ $1050 \mathrm{~kg} / \mathrm{m}^{3}$ and a dynamic viscosity, $\mu=3.45 \times 10^{-3}$
Pa.s for the flow field computation.

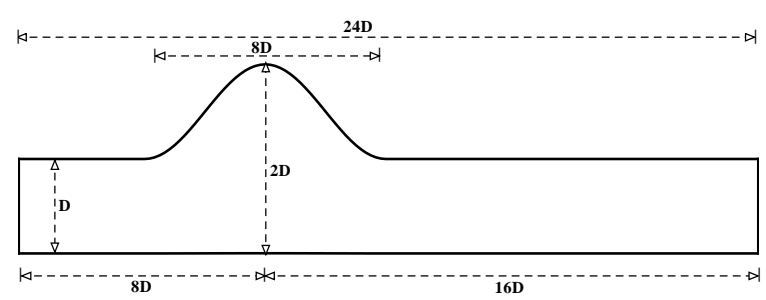

Figure 1. Geometry used in the study in the asymmetric case.

The geometry of the abdominal aorta with single aneurysm dilated asymmetrically on one side of the central axis is shown in figure (1). The aorta upstream and downstream to the AAA has been considered as a straight rigid channel. Due to the presence of the aneurysm, the height of the channel, $\delta$, is a variable in the streamwise direction (i.e., $\delta=\delta(x)$ ). Away from the aneurysm, the height of the channel is constant and is represented here using $D$ (i.e., $\delta=D$ ). The aneurysm is centered at $8 \mathrm{D}$ downstream from the channel inlet (i.e., the inlet location is $x / D=-8$ ) and $16 D$ upstream from the channel outlet where the length of the aneurysm is considered to be $8 D$. The length of the pre-aneurysm region has been considered to be smaller than the postaneurysm region for computational convenience. The cosine shaped asymmetric dilated region is modeled using the following formula :

$$
\frac{y}{D}=1+\frac{f_{c}}{2}\left[1+\cos \left(\frac{\pi x}{4 D}\right)\right] ; \quad-4 D \leq x \leq 4 D
$$

Where, $f_{c}=\delta / D$ is a parameter that controls the degree of dilation of the aneurysm. In this study, $f_{c}=1$, which results in a $100 \%$ dilation of the cross-sectional area at the center of the aneurysm.

\section{Governing Equations and Boundary Conditions}

Numerical simulation have been performed to evaluate different variables that indicate the AAA rupture risk in this study. The Navier-Stokes equations in Cartesian curvilinear coordinate system have been employed which governs the flow field as

$$
\frac{1}{|\mathbf{J}|}\left[A_{11} \frac{\partial u}{\partial \xi_{1}}+A_{12} \frac{\partial u}{\partial \xi_{2}}+A_{21} \frac{\partial v}{\partial \xi_{1}}+A_{22} \frac{\partial v}{\partial \xi_{2}}\right]=0
$$

$$
\begin{gathered}
\rho \frac{\partial u}{\partial t}+\frac{\rho}{|\mathbf{J}|}\left[u A_{11}+v A_{21}\right] \frac{\partial u}{\partial \xi_{1}}+\frac{\rho}{|\mathbf{J}|}\left[u A_{12}+v A_{22}\right] \frac{\partial u}{\partial \xi_{2}} \\
=-\left[\frac{A_{11}}{|\mathbf{J}|} \frac{\partial p}{\partial \xi_{1}}+\frac{A_{12}}{|\mathbf{J}|} \frac{\partial p}{\partial \xi_{2}}\right]+\frac{\mu}{|\mathbf{J}|^{\mathbf{2}}} \frac{\partial^{2} u}{\partial \xi_{1}{ }^{2}}\left(A_{11}^{2}+A_{21}^{2}\right)+ \\
2 \frac{\mu}{|\mathbf{J}|^{\mathbf{2}}} \frac{\partial^{2} u}{\partial \xi_{1} \partial \xi_{2}}\left(A_{11} A_{12}+A_{21} A_{22}\right)+\frac{\mu}{|\mathbf{J}|^{\mathbf{2}}} \frac{\partial^{2} u}{\partial \xi_{2}{ }^{2}} \\
\left(A_{12}^{2}+A_{22}^{2}\right)
\end{gathered}
$$




$$
\begin{array}{r}
\rho \frac{\partial v}{\partial t}+\frac{\rho}{|\mathbf{J}|}\left[u A_{11}+v A_{21}\right] \frac{\partial v}{\partial \xi_{1}}+\frac{\rho}{|\mathbf{J}|}\left[u A_{12}+v A_{22}\right] \frac{\partial u}{\partial \xi_{2}} \\
=-\left[\frac{A_{21}}{|\mathbf{J}|} \frac{\partial p}{\partial \xi_{1}}+\frac{A_{22}}{|\mathbf{J}|} \frac{\partial p}{\partial \xi_{2}}\right]+\frac{\mu}{|\mathbf{J}|^{2}} \frac{\partial^{2} v}{\partial \xi_{1}{ }^{2}}\left(A_{11}^{2}+A_{21}^{2}\right)+ \\
2 \frac{\mu}{|\mathbf{J}|^{2}} \frac{\partial^{2} v}{\partial \xi_{1} \partial \xi_{2}}\left(A_{11} A_{12}+A_{21} A_{22}\right)+\frac{\mu}{|\mathbf{J}|^{2}} \frac{\partial^{2} v}{\partial \xi_{2}{ }^{2}} \\
\left(A_{12}^{2}+A_{22}^{2}\right)
\end{array}
$$

Boundary Conditions The boundary conditions for the velocity components $u$ and $v$ are imposed as follows : (1) fully developed time varying parabolic profile at the inlet, $u(y, t)=6 U(y / D)(1-y / D)(1+0.1 \sin (t))$; (2) no-slip at both walls and (3) zero gradient outflow condition at the outlet where $\frac{\partial u}{\partial x}=0$ and $\frac{\partial v}{\partial x}=0$.

\section{Numerical Procedures}

A FORTRAN computer code is used in present computational study where finite volume approach is used to discretise the Navier-Stokes equations into a system of algebraic equations. To facilitate computation, the governing equations are transformed into curvilinear coordinates.

\subsection{Coordinate Transformation}

Thomson [15] introduced an approach where the equations are formulated in a transformed curvilinear coordinate system that coincides with the boundaries of the fluid domain. In this approach flow domain in physical space is mapped onto a rectangular domain in computational space, as shown in Figure 2 and 3, respectively. For mapping $x_{j} \longrightarrow \xi_{j}$ if $J_{i j}$ represents the elements of the Jacobian matrix, J, of the transformation then

$$
J_{i j}=\frac{\partial x_{i}}{\partial \xi_{j}}
$$

The determinant of the Jacobian matrix, $\mathbf{J}$ is denoted by $|\mathbf{J}|$ and given by

$$
|\mathbf{J}|=\frac{\partial x_{i}}{\partial \xi_{j}} A_{i j}
$$

where $A_{i j}$ are the elements of the cofactor matrix, $\mathbf{A}$, of the jacobian, defined as

$$
|\mathbf{A}|=|\mathbf{J}| \mathbf{J}^{-1}
$$

Applying the chain rule, the derivatives can now be expressed in the transformed variables in the following way

$$
\frac{\partial \phi}{\partial x_{i}}=\frac{\partial \phi}{\partial \xi_{j}} \frac{\partial \xi_{j}}{\partial x_{i}}=\frac{A_{i j}}{|\mathbf{J}|} \frac{\partial \phi}{\partial \xi_{j}}
$$

where $\phi$ is a generic variable.

A three point backward difference formula is used for time derivation of the velocity where the central difference is used for the convective and diffusion terms. Here a pressure correction algorithm is used where pressure

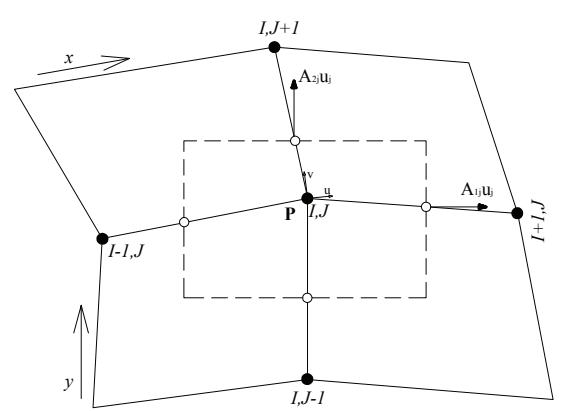

Figure 2. Grid arrangement and notation in two-dimensional case in both physical space. Solid lines and the dashed lines indicate the grid lines and the faces of the control volume respectively.

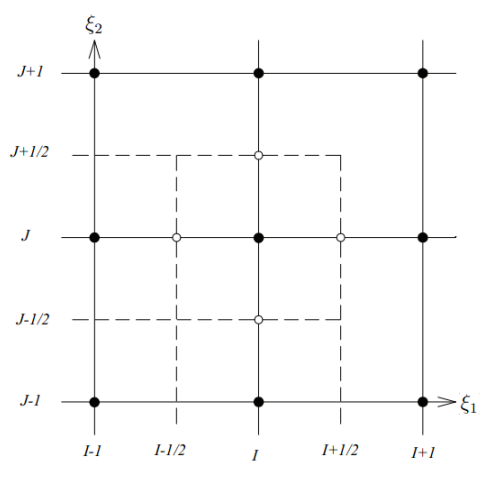

Figure 3. Grid arrangement and notation in computational space.

as well as the velocity components are stored at the center of a control volume according to the collocated grid arrangement. In our study, the grid is generated by algebraic mapping which reflects the curvilinear coordinates. Uniformity is maintained in the stream wise direction whereas the grid node spacing is non-uniform in the cross stream direction using the tan hyperbolic function to produce dense mesh near the walls to achieve more accuracy in results.

The Poisson like pressure correction equation is discretised using the pressure smoothing approach, which prevents the even-odd node uncoupling in the pressure and velocity fields. A BI-CGSTAB(Bi-Conjugate Gradient Stabilized) [5] solver is used for solving the matrix of velocity vectors, while for the Poisson like pressure correction equation a ICCGSTAB (Incomplete Cholesky Conjugate Gradient Stabilized) [18] solver is applied due to its symmetric and positive definite nature. Overall the code is second order accurate in both time and space.

\section{Result and Discussion}

A detailed description about a set of hemodynamic factors like wall shear stress, wall pressure, centerline velocity in streamwise direction, streamlines or vortex dynamics can give a better understanding about the relationship between the fluid dynamics in pulsatile blood flow and arterial disease like aneurysm. In this section, plots are demonstrated that show the results of the numerical investigation about the variables that largely contribute to the growth, development and sometimes rupture of an abdominal aortic aneurysm (AAA). Numerically simulated results are also obtained for shear 
stress and vortex formation scenario at different phases of a sinusoidal cycle ranging from 0 to $2 \pi$ for Reynolds numbers 100, 300 and 500 .

\subsection{A. Grid Independent Test}

This method attempts to find the same fundamental solution independent of grid size; this ability to retain consistency across varying grid sizes is termed as 'grid independence'. Here, different combination of grids has been chosen as different cases that are -

Case $1:(181 \times 71) \approx(x \times y) ; \quad$ Case $2:(151 \times 81) \approx$ $(x \times y)$ and Case $3:(181 \times 91) \approx(x \times y)$

In this section, the grid independence test is performed for the stream wise velocity at different locations for above three cases considering $\mathrm{Re}=500$ in an asymmetric model aneurysm. In Figure 4, it is observed that results for all three cases converge to almost same solution throughout the artery having a bit variation from frames 4(e) to 4(i). These five frames demonstrate the positions that are very close to the center of the aneurysm having the largest area. Again, the largest variation in the results is observed for three cases at the center of the bulge where Case 1 shows the velocity of 1.5, Case 2 shows 1.85 and Case 3 shows 2.05 in frame $4(\mathrm{~g})$.

It is also noticed here that Case 2 and Case 3 show very close results in all the stream wise locations for shreamwise velocities in comparison to Case 1. Therefore, it is convenient to investigate the characteristics of the hemodynamic factors for Case 2 or 3 as they provide very appropriate and convergent solutions and Case 2 has been chosen in this work to study the predicting parameters. However, it can be concluded that the present grid is capable of providing convergent solution independent of different grid sizes since the highest variation in this study is also in a negligible scale.
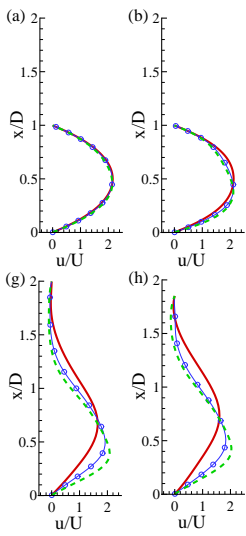

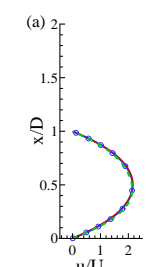

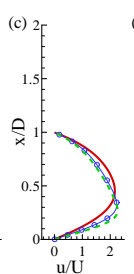
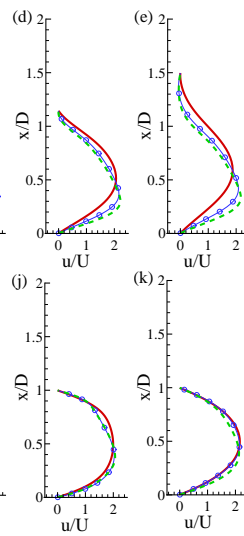

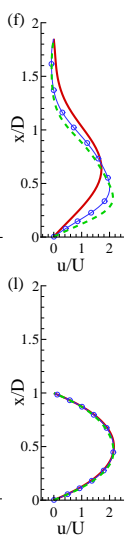

Figure 4. Grid independence test for $u / U$ in an asymmetric shaped aneurysm on the axial locations a) inlet, (b) $x / D=$ $-8.0,(\mathrm{c}) x / D=-4.0,(\mathrm{~d}) x / D=-3.0,(\mathrm{e}) x / D=-2.0$, (f) $x / D=-1.0$, (g) $x / D=0.0,(\mathrm{~h}) x / D=1.0,(\mathrm{i}) x / D=2.0,(\mathrm{j})$ $x / D=4.0,(\mathrm{k}) x / D=8.0,(\mathrm{l})$ outlet where Case $1:$ solid lines, Case 2 : dotted lines and Case 3 : circles.

\subsection{Wall shear stress (WSS)}

The flow pattern is dependent on the friction within the fluid and between the fluid and the vessel wall. This friction creates a tangential force exerted by the flowing fluid on the surface of the artery and is referred to as the "wall shear stress" which is defined as $\tau=\left.\mu \frac{\partial u}{\partial y}\right|_{\text {wall }}$ where, $\mu$ is fluid viscosity, $\frac{\partial u}{\partial y}$ is the shear rate or the velocity gradient at the wall (change in velocity unit per change in radial distance unit), $u$ is the velocity along the vessel axis, and $y$ is the distance perpendicular to the wall.

Highly oscillatory WSS at the arterial wall causes the highest risk for the development of atherosclerosis [13] and also the development of stenosis in the region downstream from the aneurysm [7]. So, it is very important to study the nature of WSS inside an aneurysm. The behavior of WSS in an artery with an asymmetric model aneurysm is described here.

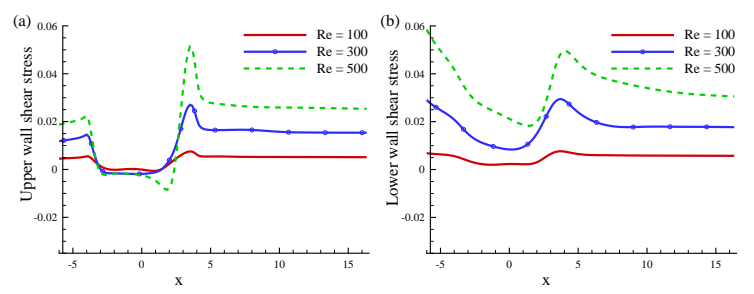

Figure 5. (a) Upper wall shear stress (WSS) and (b) lower wall shear stress (WSS) distribution for $R e=100,300$ and 500 .

Figure 5(a)-(b) show WSS respectively for upper wall and lower wall for an asymmetric shaped aneurysm where both of them designate nearly similar characteristics. The upper and lower wall shear stress is characterized by an oscillatory behavior during the pulsatile cycle. All three Reynolds numbers demonstrate a constant shear stress throughout the pre-aneurysm zones. After that, at the proximal neck of the aneurysm there is a drop in WSS having the lowest magnitude near the center of the aneurysm at $x=1.9$. The highest shear stress occurs at the distal neck which may be due to the local acceleration of the flow. This is followed by again a constant pattern in the post-aneurysm region for all three Reynolds numbers. The patterns of WSS in different points of the artery found here match with the experimental results by Egelhoff et al. [1].

Although the level of shear stress is very low (around zero) in each case, there is a small variation in the lower WSS from the upper WSS due to the shape of the model. The upper WSS is very small, near zero around the inlet position but the lower WSS is higher than zero at the inlet which gradually decreases through the preaneurysm zone. The range of variation for upper WSS is $(-0.006,0.046)$ while for lower WSS, it is $(0,0.059)$.

WSS falls inside the aneurysm because of the increasing area which leads to a decrease in velocity of the flow. Another key point is that greater Reynolds number causes higher values of wall stress and vice versa. Since higher Reynolds number causes turbulence in fluid dynamics, it increases the velocity as well as the wall shear stress.

The WSS through an arterial aneurysm of asymmetric shape for $\mathrm{Re}=100,300$ and 500 considering different phases of a cardiac cycle has also been evaluated in this work which is depicted in Figure 6 and 7. These WSS diagrams are illustrated based on a sinusoidal cycle which is equivalent of a cardiac cycle shown in frame 6(a). 
Eleven distinct cases are plotted here for WSS where each case represents a particular phase of the sinusoidal cycle which is also shown inset.
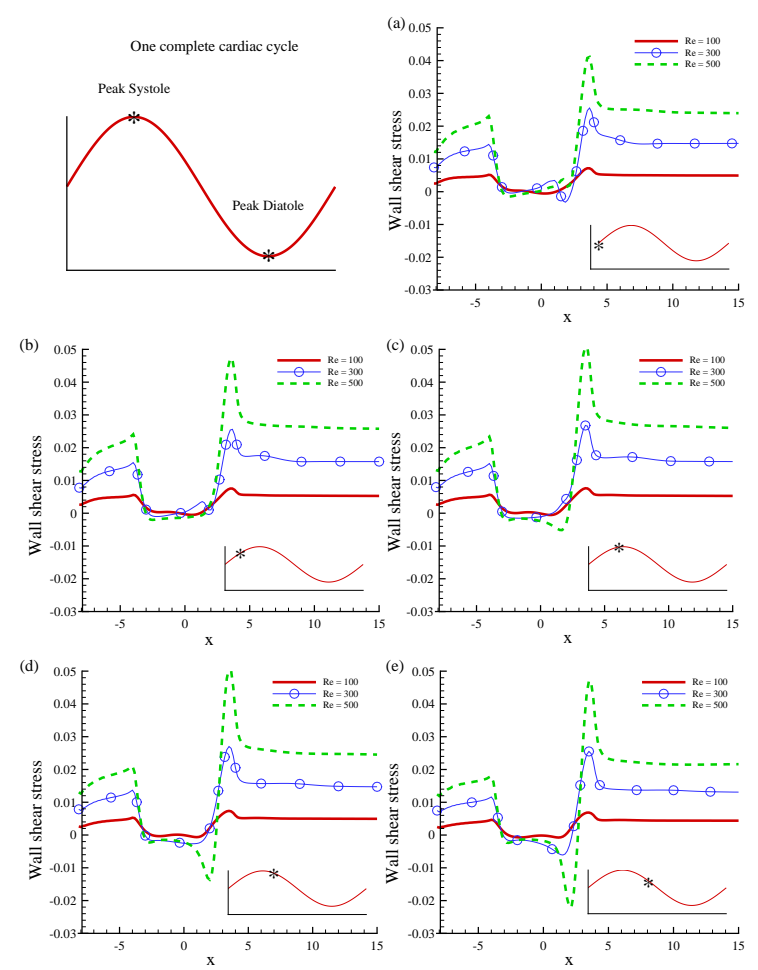

Figure 6. Upper wall shear stress (WSS) distribution for the flow in an asymmetric shaped aneurysm at the different pulsatile phases (a) $t / T=9.0,(\mathrm{~b}) t / T=9.1,(\mathrm{c}) t / T=9.2$, (d) $t / T=9.3,(\mathrm{e})$ $t / T=9.4$ (Continue...)

At a glance over the diagram, it is clearly seen that all the Reynolds numbers follow a parallel pattern in the pre and post-aneurysm regions during the entire cycle. The WSS has a very small value swing between $(-0.026,0.049)$ which falls down inside the aneurysm for all the Reynolds numbers. A constant WSS is found for three Reynolds numbers in the pre-aneurysm region. At $t / T=9.0$ and $t / T=9.1$, it drops for all the Reynolds numbers by equal amount but in the second half of the systolic phase and also in early diastole the WSS drops for $R e=300$ and 500 to a larger extent. The lowest WSS with a value -0.026 at $t / T=9.5$ for $R e=500$ is observed near the center of the aneurysm. It again starts increasing inside the aneurysm bulge in the following phases and the WSS pattern of the late diastole completely matches with the early systolic one.

The peak WSS is observed at the distal end of the bulge due to the local acceleration of the flow. The highest value of WSS is found to be 0.049 at the peak systole caused by $R e=500$ shown in frame $6(\mathrm{~d})$ at $t / T$ $=9.3$ which again drops to a small extent in the following phases. The shear stress also rises at $t / T=10$ to catch the similar pattern as the early systolic phase. Throughout the post-aneurysm region the WSS follows a constant value that maintains a parallel pattern for the three Reynolds numbers.

\subsection{Pressure field}

Arterial wall pressure is one of the important indicating factors to predict the risk caused by the development of
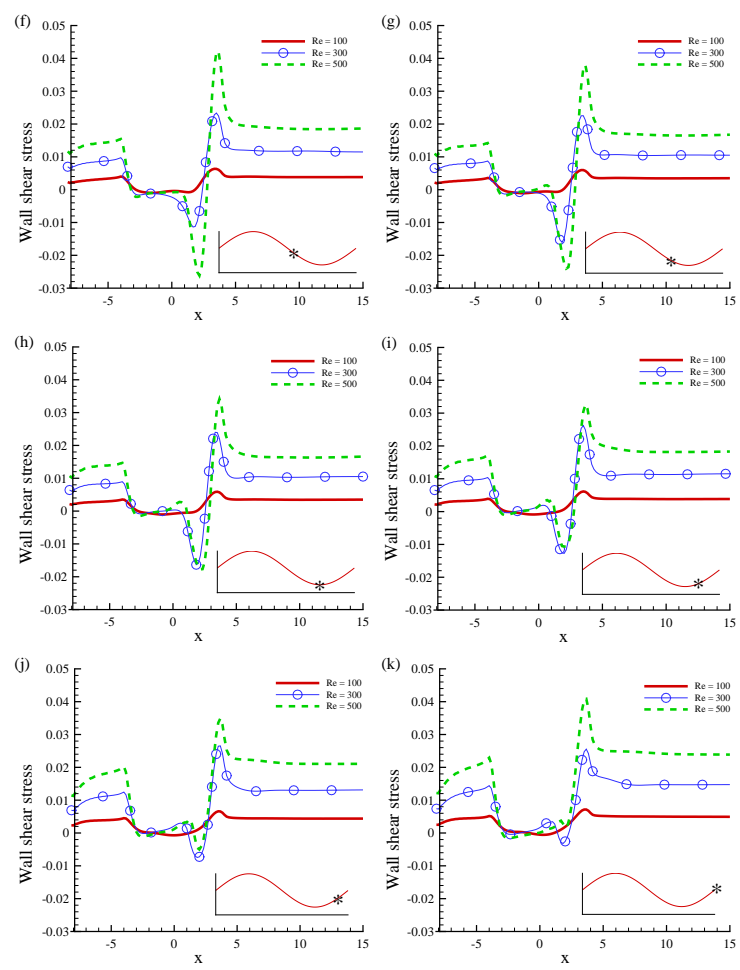

Figure 7. Upper wall shear stress (WSS) distribution for the flow in an asymmetric shaped aneurysm at the different pulsatile phases (f) $t / T=9.5$, (g) $t / T=9.6$, (h) $t / T=9.7,(\mathrm{i}) t / T=9.8,(\mathrm{j})$ $t / T=9.9,(\mathrm{k}) t / T=10$

aortic aneurysm.

Figure 8 shows upper wall pressure variation in an asymmetric model aneurysm. The wall pressure varies the most and follows a zigzag pattern for the $\mathrm{Re}=500$ in this case. The figure shows less variations in wall pressure for the flow with $\mathrm{Re}=300$ and almost smooth pattern for $\operatorname{Re}=100$. The temporal accelerations and decelerations of the flow largely influence the pressure distribution at the arterial wall during the pulsatile flow cycle.

The flow produces almost constant pressure in the preaneurysm regions but it drops suddenly down just at the proximal neck of the bulge. Then it starts increasing and follows a rise-drop pattern inside the aneurysm and attains the largest value at the distal end. In the post-aneurysm region the wall pressure starts decreasing gradually. In this asymmetric geometry, greater Reynolds number causes less pressure and the smaller one causes more wall pressure. Since greater Reynolds number causes higher velocity and according to the Bernoulli equation decreasing velocity causes increasing pressure, flow maintains an inversely proportional relationship between the wall pressure and the Reynolds number. Another important observation is -the wall pressure always maintains negative value for all three Reynolds numbers in this asymmetric geometry. The diverging - converging shape of the artery seems to have an effect on the pressure distribution.

While investigating the lowerwall pressure of the artery with an asymmetric shaped aneurysm in Figure 9, nice similarity is observed between the upper wall and lower wall pressure distribution in this geometry. Here the flow with $\operatorname{Re}=500$ experiences more variations than the other two Reynolds numbers. There is also a rise- 

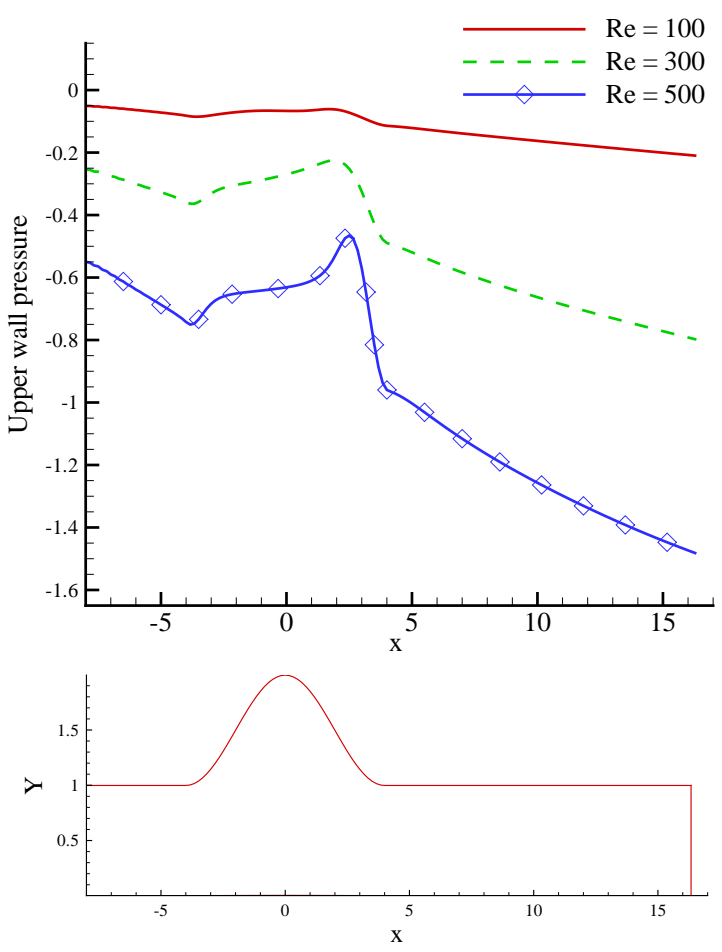

Figure 8. Upper wall pressure, $\left(p / \rho U^{2}\right)$ distribution for $\mathrm{Re}=$ 100,300 and 500 in an asymmetric shaped aneurysm.
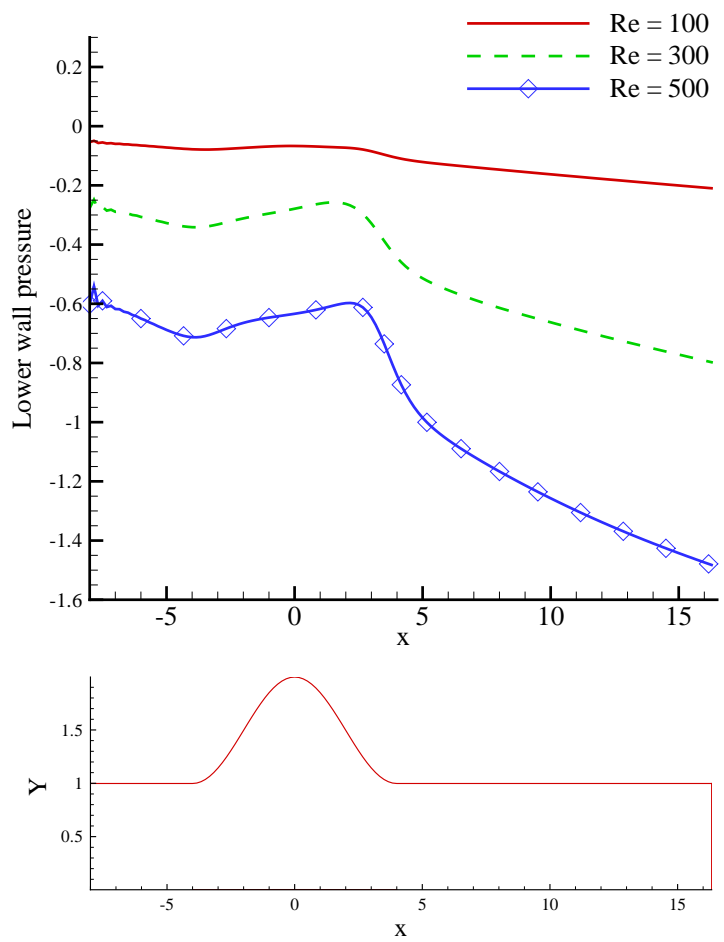

Figure 9. Lower wall pressure, $\left(p / \rho U^{2}\right)$ distribution for $\operatorname{Re}=$ 100,300 and 500 in an asymmetric shaped aneurysm. drop pattern in wall pressure inside the aneurysm having the peak value at the distal end of the bulge. Only difference is- the variations in pressure distribution inside the bulge is less than upper wall pressure. Unlike the upper wall pressure the lower wall pressure follows a parallel pattern for all the Reynolds numbers where $R e=500$ has the lowest and $R e=100$ has the largest range of pressure variation. Nevertheless, all the Reynolds numbers show indifferent characteristics inside the bulge i.e., they have the peak value near the distal end and then again a constant pattern.

The arterial vessel structure depends on the balance between the intraluminal pressure and the wall's ability to resist the pressure. If any elongation of the arterial wall occurs due to the presence of aneurysm, the intraluminal pressure may overcome the wall's ability to resist and may weaken the wall by further elongating it [7]. Higher wall pressure found inside the aneurysm might account for the extra intraluminal pressure and thus further weakening of the wall.

\subsection{Central line velocity}

Studying the flow intensity inside an aneurysm can help to predict the rupture risk of the dilated area, magnitude of velocity at every point inside it is an important indicator as well. In the following part the results of centerline velocity caused by the pulsatile flow in an abdominal aorta with aneurysm is going to be discussed.

Figure 10 shows the center line velocity caused by the flows with Reynolds numbers 100, 300 and 500 through an asymmetric model aneurysm. It can easily be observed that the centerline velocity does not follow exactly constant pattern from the inlet position throughout the pre-aneurysm region in the asymmetric case rather it starts increasing gradually after the inlet position up to the proximal neck of the bulge. As the flow enters the bulge it decreases gradually having the lowest peak near the center of the dilated region. It drops down inside the aneurysm for all three Reynolds numbers which can be explained by the Bernoulli equation and the one-dimensional continuity equation. The centerline velocity follows again an almost constant pattern in the post-aneurysm regions. From this Figure 10, it can be clearly seen that the velocity varies within a range $(0.8,1.2)$ for $\operatorname{Re}=500,(0.6,1.2)$ for $\operatorname{Re}=300$ and $(0.25,0.49)$ for $R e=100$. Hence, higher Reynolds numbers produce centerline velocities with higher values and vice versa. It proves the linear relationship between the Reynolds number and velocity caused by pulsatile flow.

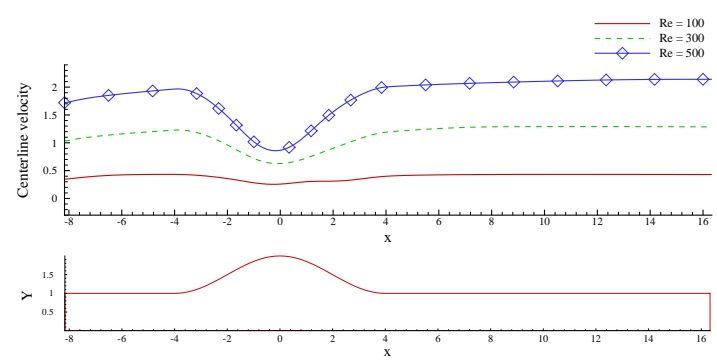

Figure 10. Centerline velocity, $u / U$ profile for $R e=100,300$ and 500 . 


\subsection{Streamwise velocity}

Here, the velocity profiles that are present at different positions throughout the arterial segment with aneurysm are explored. The stream wise velocity in an artery with an asymmetric shaped aneurysm is shown in Figure 11(a).

The result of streamwise velocity flow through an arterial aneurysm is represented here for three distinct Reynolds numbers. The investigation has been started with the inlet position and end with outlet but between these two positions several points were also taken to show how velocity develops gradually inside the artery in these points. The $x$-axis represents the velocity profile in some stream wise locations where the $y$-axis shows different locations on the artery. A nice parabolic shape of the velocity is observed in the inlet which is shown in frame 11(a). From frames 11(b) and 11(c), the velocity pattern through the artery is observed in the preaneurysm regions where the magnitudes of the stream wise velocities for different Reynolds numbers increase in an infinitesimal level. The velocity profile here gradually loses its natural symmetric behavior. Frame 11(d) represents the velocity in the proximal end of the aneurysm where the peak occurs in the lower portion of the artery which has a value 2.2 for $R e=500$. The velocity pattern exactly at the center of the dilated region can be approximated from frame $11(\mathrm{~g})$ which indicates a drop in velocity for all Reynolds numbers. Inside the aneurysm from frame $11(\mathrm{~h})$ to $11(\mathrm{i})$ the velocity pattern is almost same where they are entirely deviated from the inlet parabolic shape. The lowest velocity is also approximated at the distal end of the aneurysm having a value of 1.973 that is indicated by frame $11(\mathrm{j})$. Through the post-aneurysm region the velocity profile starts increasing gradually. Frame 11(o) shows the outlet velocity which completely matches with the inlet one due to the effect of re-laminarization.

In this asymmetric shaped aneurysm, the flow gets a wider region inside the aneurysm on the upper side of the artery while there is no extended area toward the lower portion. According to the Bernoulli equation the velocity decreases in the upper portion and increases in the lower portion here. The velocity profiles also follow a linear relationship with the Reynolds numbers as the presence of any vortex is not observed for $R e=100$.

\subsection{Streamlines}

Figure 12 contains the streamlines of the pulsatile flow in a $100 \%$ dilated vessel, respectively, for three different Reynolds number $\mathrm{Re}=100,300$ and 500. Frame 12(a) shows streamlines for $\mathrm{Re}=100$ where the flow is partially developed inside the dilated region that helps to form small recirculation zones under the extended part of the vessel. This vortex tends to become fatter and longer with the increasing values of Reynolds number. Larger vortex is observed if the Reynolds number is increased to 300 which covers the whole dilated portion. Since the flow becomes fully developed, the vortex rings nestled in the concavity of aneurysm grow in size filling most of the dilated portion of the vessel in frame 12(c) which is caused by the flow with $\operatorname{Re}=500$.
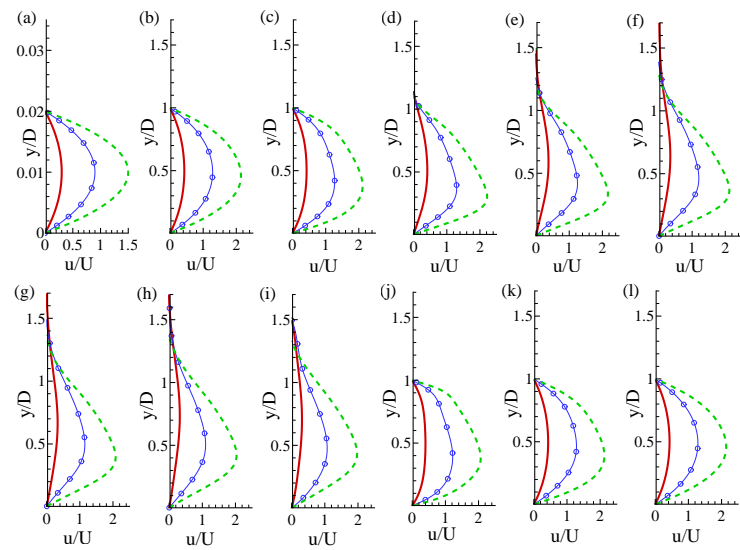

Figure 11. Streamwise velocity $u / U$, at the different axial position a) inlet, (b) $x / D=-8.0$,(c) $x / D=-4.0$, (d) $x / D=$ $-3.0,(\mathrm{e}) x / D=-2.0$, (f) $x / D=-1.0$, (g) $x / D=0.0,(\mathrm{~h})$ $x / D=1.0,(\mathrm{i}) x / D=2.0,(\mathrm{j}) x / D=4.0,(\mathrm{k}) x / D=8.0,(\mathrm{l})$ outlet where solid lines represent $R e=100$, lines with circles represent $R e=300$ and dotted lines represent $R e=500$.

The Reynolds number is defined by the ratio of inertial effects to viscous effects in the flow. As the viscosity is constant in this study, the Reynolds number maintains linear relationship with the inertial effects in the flow. For large Reynolds numbers inertial effects are dominant which causes translational behavior in the flow while small Reynolds numbers always cause laminar flow behavior. Since some sort of chaotic motions are observed in the flow pattern, it can be concluded that translational nature is found in flow dynamics for cosine shaped dilated geometry even for $\mathrm{Re}=100$ in this work.

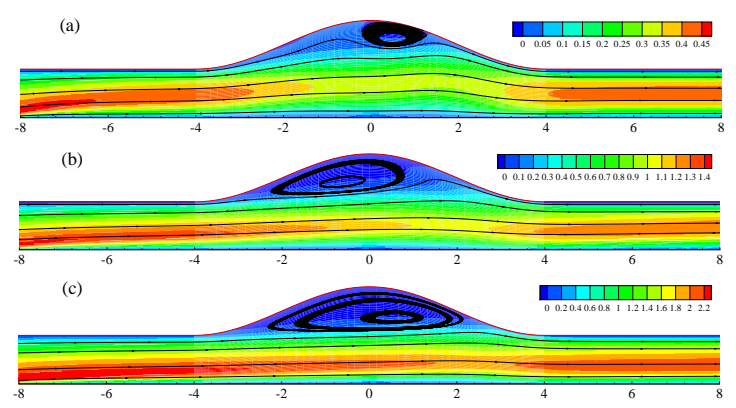

Figure 12. Streamlines inside an asymmetric shaped aneurysm for (a) $R e=100$ (b) $R e=300$ and (c) $R e=500$.

\subsection{Recirculation zones}

The behavior of flow dynamics in an artery with an asymmetric shaped aneurysm is depicted in Figure 13 and 14. Here, the upper wall of the artery is extended outward to some extent and the lower portion of it has been considered to be unchanged. The flow is laminar through the entire cycle in the lower portion of the artery. For $t / T=9.0$ a small recirculation zone is seen in the distal end of the aneurysm. The recirculation region shifts to the proximal end of the aneurysm at $t / T=9.1$. Through 13(c) and 13(d) the vortex becomes larger in the proximal end part but covers almost the entire dilated portion through the first half of the diastole shown in $13(\mathrm{e}), 13(\mathrm{f})$ and $14(\mathrm{~g})$. The largest vortex in $14(\mathrm{~g})$ found is during the first half of the diastolic phase. From 
frames $14(\mathrm{~h})$ to $14(\mathrm{j})$ it is observed that the vortex shifts to distal end and gradually becomes smaller. Through phases $t / T=9.8$ to $t / T=10$, the strength of turbulence decreases steadily and the nature of flow for $t / T=10$ becomes closely same as $t / T=9.0$. In other words, the flow pattern becomes the same as the initial phase after completing one full cycle.

In short, it can be said that flow is laminar around the central axis in an artery with an asymmetric shaped aneurysm. However, there is chaotic motion near the wall of the dilated regions that leads to increase the recirculation area. The vortex is shifted from the distal end to the proximal end and again to the distal end within single cardiac pulse. Eventually, the highest translational nature is found in the first half of the diastole.

The effect of asymmetry on flow patterns is significant irrespective of aneurysm size. Larger asymmetric aneurysms are more prone to increased wall stress distributions, high WSS values and increased overall pressure in the bulge and hence are more likely to rupture. The rupture risk for large asymmetric aneurysm is highest at the posterior distal end of the aneurysm bulge due to the complex flow patterns in this region[11].

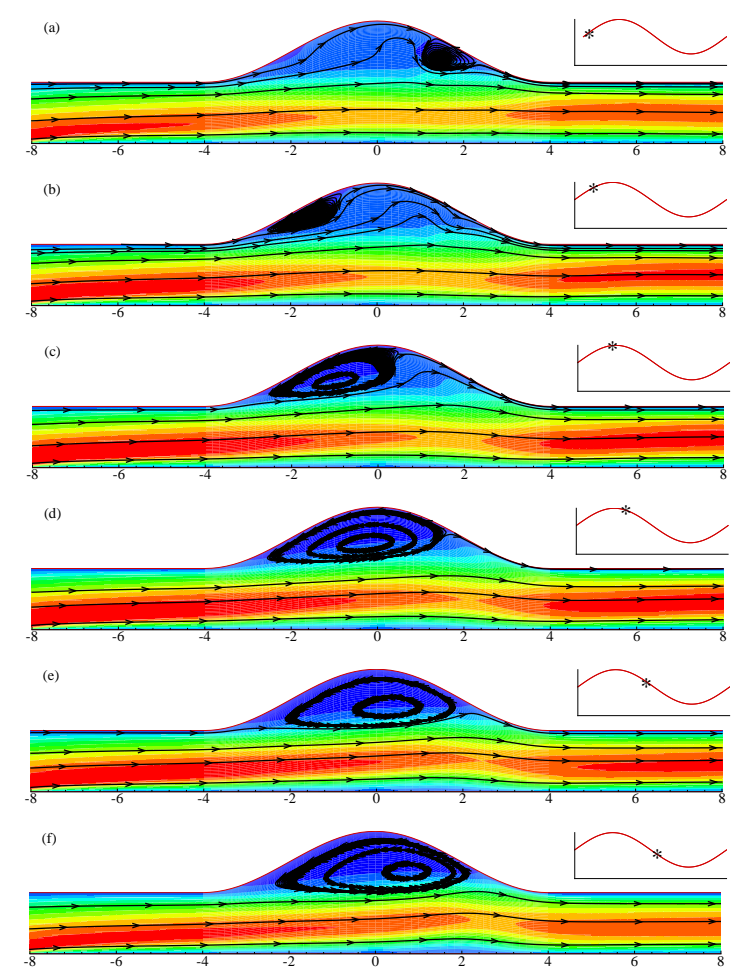

Figure 13. Vortex dynamics for the blood flow through an asymmetric shaped arterial aneurysm at the different pulsatile phase (a) $t / T=9.0$,(b) $t / T=9.1$,(c) $t / T=9.2$, (d) $t / T=9.3$,(e) $t / T=9.4,(\mathrm{f}) t / T=9.5$ (Continue...)

\subsection{Cycle-to-cycle velocity and pressure}

A study on the outline of stream wise velocity and pressure with respect of time has been made here. In other words how it changes with time in different positions of the artery. In these frames 1,13 and 6 represent the time history for inlet, outlet and the center point of the aneurysm, respectively.

While looking at the time history of stream wise centerline velocity for the asymmetric shaped aneurysm in
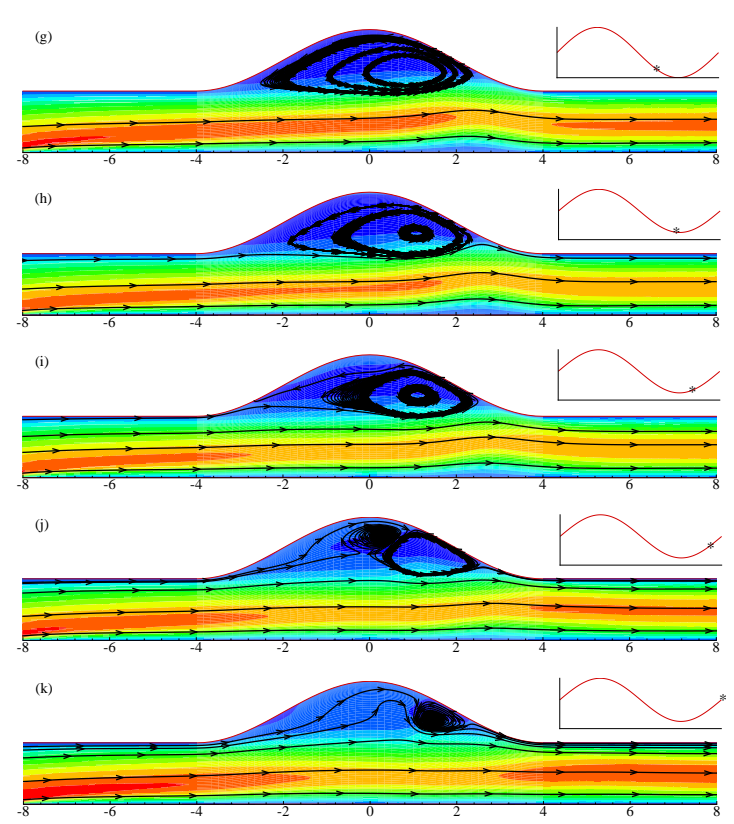

Figure 14. Vortex dynamics for the blood flow through an asymmetric shaped arterial aneurysm at the different pulsatile phase $(\mathrm{g})$ $t / T=9.6,(\mathrm{~h}) t / T=9.7$,(i) $t / T=9.8,(\mathrm{j}) t / T=9.9,(\mathrm{k}) t / T=10$

Figure 15, it is observed that the velocity in the direction of the stream is fully sinusoidal in the latter half of the cycle for all locations in the stream wise direction. Oscillation is found in velocity in the earlier time only which again varies with different stream wise locations. Inlet and outlet position provide with almost smooth sinusoids where it changes rapidly in the proximal end of the aneurysm shown by frames 15(2), (3) and (4). The velocity pattern turns into a sharp peak in the middle of the aneurysm which continues up to the distal end part. The velocity becomes damped sinusoid in the post aneurysm regions which becomes distorted sinusoidal profile at the outlet.

For the time results for pressure distribution in Figure 16, the shape is found to be closely like the stream wise velocity. However, increasing frequency, neither like sinusoids nor E.C.G, is shown at the initial stream wise locations of the time period which gradually becomes nearly straight line unlike stream wise velocity. A non chaotic pressure distribution is observed in the inlet position. Moreover, distorted sinusoids are found in frames $16(1)$ to (11) that indicate the locations from the inlet to the end of the aneurysm. From frames 16(12) to (14) a damped sinusoid is gradually developed and completed at the outlet.

\section{Conclusion}

Finite volume numerical simulation of unsteady, incompressible, Newtonian blood flow in two dimensional rigid models of $100 \%$ dilated single aneurysm have been presented herein. Flow field, flow induced wall pressure and wall shear stress have been investigated in a rigid channel with an asymmetric shaped aneurysm under pulsatile condition for $\mathrm{Re}=100,300$ and 500. The maximum shear stress is observed to occur at the distal neck of the aneurysm where the upper WSS is smaller than the lower one in the inlet position. The WSS is also 


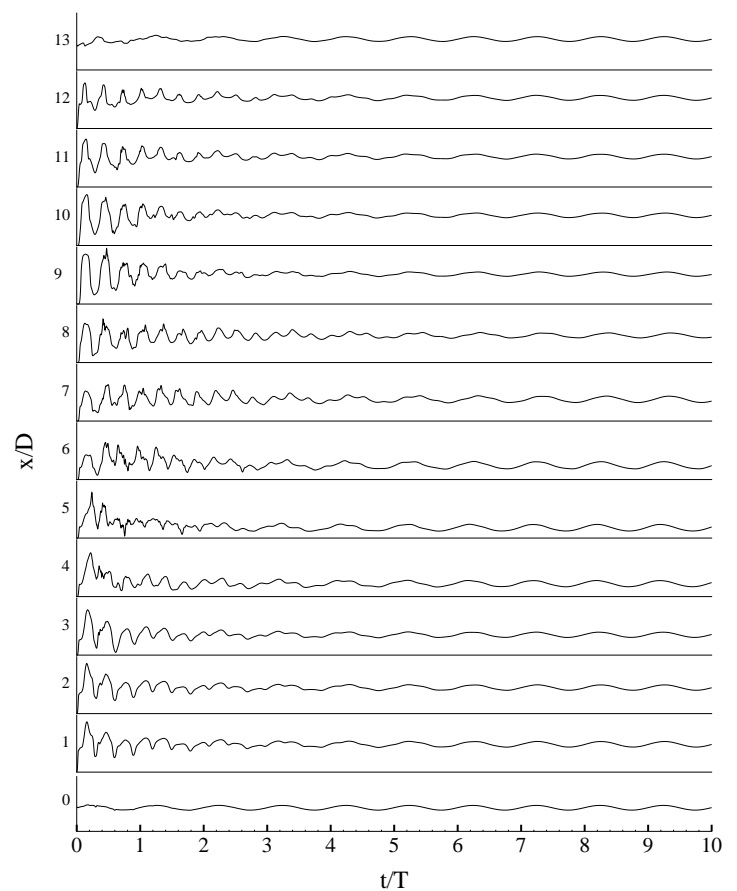

Figure 15. Time history for the stream wise velocity, $u / U$.

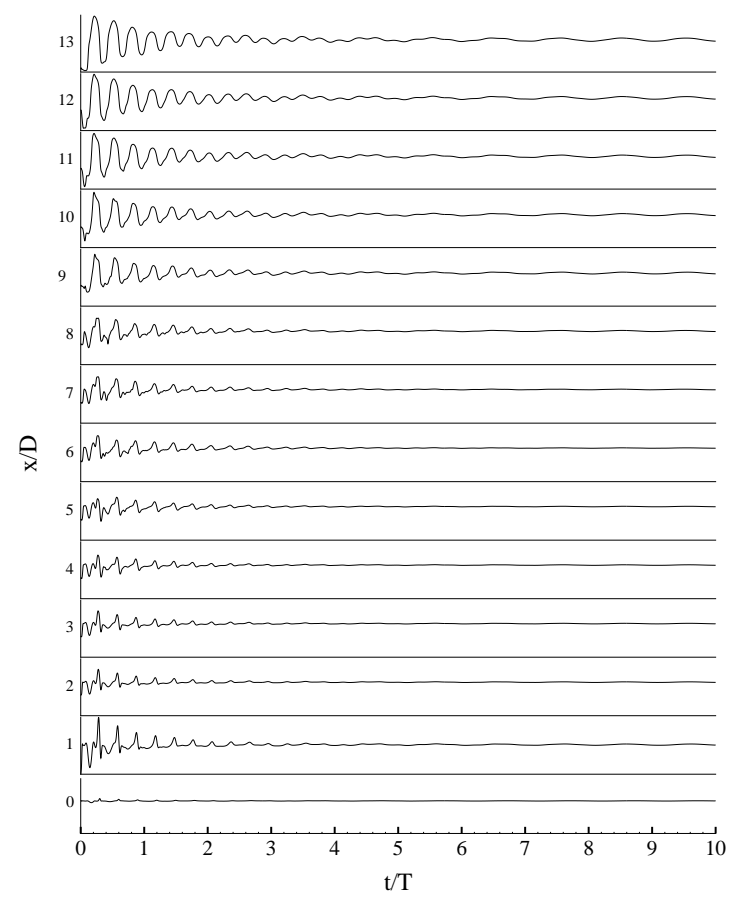

Figure 16. Time history for pressure distribution, $p / \rho u^{2}$. characterized here by means of a sequence of different flow stages in one period of the cardiac pulse. The highest value of WSS is found at the peak systole caused by $\operatorname{Re}=500$ due to the acceleration of flow while the lowest one occurs during early diastole. Hence, greater Reynolds number causes higher values of wall stress and vice versa.

Higher wall pressure can be an important reason of weakening arterial wall and thus formation of aneurysm which is found at the distal end in this geometry. Both the upper and lower wall pressure maintains a negative value throughout the artery segment having an inverse relationship with the Reynolds number. The diverging - converging shape of the artery might contribute to the wall pressure distribution in this asymmetric geometry. Centerline velocities for three different Reynolds numbers indicate the linear relationship between the Reynolds number and velocity caused by pulsatile flow. Through the arterial segment, the lowest velocity occurs at the center which can be explained by the Bernoulli equation and the one-dimensional continuity equation. Study of the velocity patterns along the stream wise direction shows the peak velocity at the proximal end and the lowest value at the distal end of the geometry.

Streamlines demonstrate the presence of recirculation zones in the flow field which is found in a very small scale for $\mathrm{Re}=100$ where the flow is partially developed inside the dilated region. However, this vortex tends to become fatter and longer with the increasing values of Reynolds number and thus larger recirculation zones are found for $R e=300$ and $R e=500$. A very interesting phenomenon of vortex dynamics is observed while investigating its characteristics for a full cardiac cycle. A small vortex forms initially which shifts from the distal to the proximal neck by a single pulse. The vortex shifts to the distal end through the entire diastolic phases. It becomes bigger and the largest vortex is found during the first half of the diastolic phase. Eventually, the highest translational nature is found in the first half of the diastole. The cycle-to-cycle time history for stream wise velocity shows extreme variation from the sinusoidal pattern throughout the artery while this result for pressure becomes damped sinusoids from the small E.C.G like patterns near the outlet positions.

In the end, it can be concluded that a higher Reynolds number has greater effects on the translational characteristics of a flow which is observed throughout our investigation for intense pressure, velocity and wall shear stress contributing to the growth of aneurysm.

\section{Acknowledgements}

The authors wish to acknowledge gratefully for funding from the North South University, Bangladesh during the period of this research.

\section{REFERENCES}

[1] C. J. Egelhoff, R. S. Budwig, D. F. Elger, T. A. Khraishi, and K. H. Johansen. Model studies of the flow in abdominal aortic aneurysms during resting 
and exercise conditions. J. Boimech., 32(12):13191329, 1999.

[2] A. Farnoush, Y. Qian, and A. Avolio. Effect of inflow on computational fluid dynamic simulation of cerebral bifurcation aneurysms. In Engineering in Medicine and Biology Society, EMBC, 2011 Annual International Conference of the IEEE, pages 1025-1028. IEEE, 2011.

[3] E. A. Finol and C. H. Amon. Blood flow in abdominal aortic aneurysms: Pulsatile flow hemodynamics. J. Biomech Engng., 123:474-484, 2001.

[4] E. A. Finol, K. Keyhani, and C. H. Amon. The effect of asymmetry in abdominal aortic aneurysms under physiologically realistic pulsatile flow conditions. J. Biomech. Engn., 125(2):207-217, 2003.

[5] D. S. Kershaw. The incomplete cholesky conjugate gradient method for the iterative solution of systems. J. Comput. Phys., 26:43-65, 1978.

[6] Y. H. Kim, P. J. VandeVord, and J. S. Lee. Multiphase non-newtonian effects on pulsatile hemodynamics in a coronary artery. Int. J. Numer. Meth. Fluids, 58(7):803-825, 2008.

[7] B. V. Kumar and K. B. Naidu. Hemodynamics in aneurysm. Comput. Biomed. Res., 29(2):119-139, 1996.

[8] B. V. R. Kumar. A space-time analysis of blood flow in a $3 \mathrm{~d}$ vessel with multiple aneurysms. Comput. Mech., 32:16-28, 2003.

[9] Zhi-Yong Li. Computed wall stress may predict the growth of abdominal aortic aneurysm. In Engineering in Medicine and Biology Society (EMBC), 2010 Annual Int. Conf. IEEE, pages 2626-2629. IEEE, 2010.

[10] S. T. R. Macsweeney. Pathogenesis of abdominal aortic aneurysm. British J. Surgery, 81:935-941, 1994.

[11] V. Patel. Impact of geometry on blood flow patterns in abdominal aortic aneurysms. PhD thesis, Rutgers University-Graduate School-New Brunswick, 2011 .
[12] K. Perktold. On the paths of fluid particles in an axisymmetrical aneurysm. J. Boimech., 20:311-317, 1986.

[13] A. M. Shaaban and A. J. Duerinckx. Wall shear stress and early atherosclerosis: A review. American J. Roentgenology, 174 (6):1657-1665, 2000.

[14] A. Swillens, L. Lanoye, J. De Backer, N. Stergiopulos, P. R. Verdonck, F. Vermassen, and P. Segers. Effect of an abdominal aortic aneurysm on wave reflection in the aorta. Biomedical Engineering, IEEE Transactions on, 55(5):1602-1611, 2008.

[15] J. F. Thomson, F. Thames, and C. Mastin. Automatic numerical generation of body-fitted curvilinear coordinate system for field containing any number of arbitrary two dimentiona bodiesl. J. Comput. Phys., 15:299-319, 1975.

[16] A. Valencia, J. Munizaga, R. Rivera, and E. Bravo. Numerical investigation of the hemodynamics in anatomically realistic lateral cerebral aneurysms. In Engineering in Medicine and Biology Society (EMBC), 2010 Annual International Conference of the IEEE, pages 2616-2621. IEEE, 2010.

[17] K. Y. Volokh and D.A. Vorp. A model of growth and rupture of abdominal aortic aneurysm, journal of biomechanics. J. Boimech., 41:1015-1021, 2008.

[18] H. A. D. Vorst. Bi-cgstab: a first and smoothly converging variant of bi-cg for the solution of the non-symmetric linear systems. SIAM J. Sci. Stat. Comput., 155:631-644, 1992.

[19] X. Xu and J. S. Lee. Application of the lattice boltzmann method to flow in aneurysm with ring-shaped stent obstacles. Int. J. Numer. Meth. Fluids, pages 1829-1849, 2008.

[20] T. H. Yip and S. C. M. Yu. Cyclic transition to turbulence in rigid abdominal aortic aneurysm models. Fluid Dyn. Res, 29:81-113, 2001. 